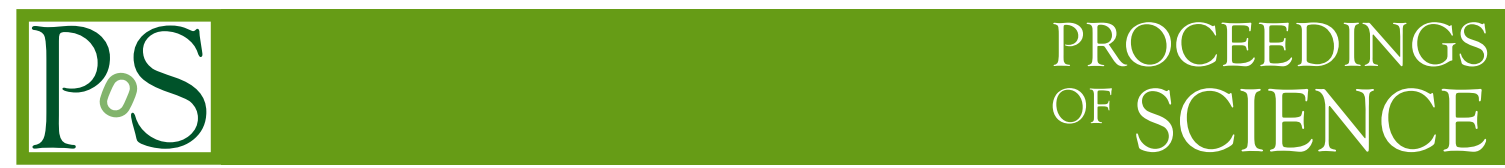

\title{
Anisotropic Flow of Strange Particles at SPS
}

\section{Grzegorz Stefanek* for the NA49 Collaboration}

Swietokrzyska Academy, Kielce, Poland

E-mail: stefanek@pu.kielce.pl

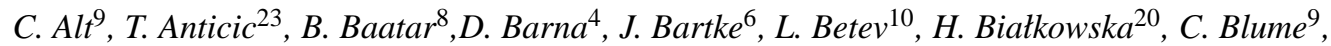
B. Boimska ${ }^{20}$, M. Botje ${ }^{1}$, J. Bracinik ${ }^{3}$, R. Bramm ${ }^{9}$, P. Bunčić ${ }^{10}$, V. Cerny ${ }^{3}$, P. Christakoglou ${ }^{2}$, P. Chung ${ }^{19}$, O. Chvala ${ }^{14}$, J.G. Cramer $^{16}$, P. Csató ${ }^{4}$, P. Dinkelaker ${ }^{9}$, V. Eckardt ${ }^{13}$, D. Flierl ${ }^{9}$, Z. Fodor ${ }^{4}$, P. Foka ${ }^{7}$, V. Friese ${ }^{7}$, J. Gál ${ }^{4}$, M. Gaździcki ${ }^{9,11}$, V. Genchev ${ }^{18}$, G. Georgopoulos ${ }^{2}$, E. Gtadysz ${ }^{6}$, K. Grebieszkow ${ }^{22}$, S. Hegyi ${ }^{4}$, C. Höhne ${ }^{7}$, K. Kadija ${ }^{23}$, A. Karev $^{13}$, D. Kikola ${ }^{22}$, M. Kliemant ${ }^{9}$, S. Kniege ${ }^{9}$, V.I. Kolesnikov ${ }^{8}$, E. Kornas ${ }^{6}$, R. Korus ${ }^{11}$, M. Kowalski ${ }^{6}$, I. Kraus ${ }^{7}$, M. Kreps ${ }^{3}$, A. Laszlo ${ }^{4}$, R. Lacey ${ }^{19}$, M. van Leeuwen ${ }^{1}$, P. Lévai ${ }^{4}$, L. Litov ${ }^{17}$, B. Lungwitz ${ }^{9}$, M. Makariev ${ }^{17}$, A.I. Malakhov ${ }^{8}$, M. Mateev $^{17}$, G.L. Melkumov ${ }^{8}$, A. Mischke $^{1}$, M. Mitrovski ${ }^{9}$, J. Molnár ${ }^{4}$, St. Mrówczyński ${ }^{11}$, V. Nicolic ${ }^{23}$, G. Pálla ${ }^{4}$, A.D. Panagiotou ${ }^{2}$, D. Panayotov ${ }^{17}$,

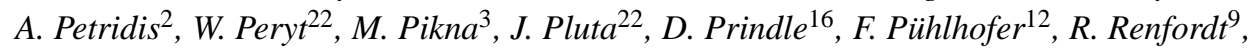
C. Roland ${ }^{5}$, G. Roland ${ }^{5}$, M. Rybczyński ${ }^{11}$, A. Rybicki ${ }^{6,10}$, A. Sandoval ${ }^{7}$, N. Schmitz ${ }^{13}$, T. Schuster ${ }^{9}$, P. Seyboth ${ }^{13}$, F. Siklér ${ }^{4}$, B. Sitar ${ }^{3}$, E. Skrzypczak ${ }^{21}$, M. Slodkowski ${ }^{22}$, G. Stefanek ${ }^{11}$, R. Stock ${ }^{9}$, C. Strabel ${ }^{9}$, H. Ströbele ${ }^{9}$, T. Susa ${ }^{23}$, I. Szentpétery ${ }^{4}$, J. Sziklai $^{4}$, M. Szuba ${ }^{22}$, P. Szymanski ${ }^{10,20}$, V. Trubnikov ${ }^{20}$, D. Varga ${ }^{4,10}$, M. Vassiliou ${ }^{2}$, G.I. Veres ${ }^{4,5}$, G. Vesztergombi ${ }^{4}$, D. Vranić ${ }^{7}$, A. Wetzler ${ }^{9}$, Z. Wtodarczyk $^{11}$, A. Wojtaszek ${ }^{11}$, I.K. Yoo ${ }^{15}$, J. Zimányi $^{4}$ 
${ }^{1}$ NIKHEF, Amsterdam, Netherlands.

${ }^{2}$ Department of Physics, University of Athens, Athens, Greece.

${ }^{3}$ Comenius University, Bratislava, Slovakia.

${ }^{4}$ KFKI Research Institute for Particle and Nuclear Physics, Budapest, Hungary.

${ }^{5}$ MIT, Cambridge, USA.

${ }^{6}$ Institute of Nuclear Physics, Cracow, Poland.

${ }^{7}$ Gesellschaft für Schwerionenforschung (GSI), Darmstadt, Germany.

${ }^{8}$ Joint Institute for Nuclear Research, Dubna, Russia.

${ }^{9}$ Fachbereich Physik der Universität, Frankfurt, Germany.

${ }^{10}$ CERN, Geneva, Switzerland.

${ }^{11}$ Institute of Physics Świȩtokrzyska Academy, Kielce, Poland.

${ }^{12}$ Fachbereich Physik der Universität, Marburg, Germany.

${ }^{13}$ Max-Planck-Institut für Physik, Munich, Germany.

${ }^{14}$ Institute of Particle and Nuclear Physics, Charles University, Prague, Czech Republic.

${ }^{15}$ Department of Physics, Pusan National University, Pusan, Republic of Korea.

${ }^{16}$ Nuclear Physics Laboratory, University of Washington, Seattle, WA, USA.

${ }^{17}$ Atomic Physics Department, Sofia University St. Kliment Ohridski, Sofia, Bulgaria.

${ }^{18}$ Institute for Nuclear Research and Nuclear Energy, Sofia, Bulgaria.

${ }^{19}$ Department of Chemistry, Stony Brook Univ. (SUNYSB), Stony Brook, USA.

${ }^{20}$ Institute for Nuclear Studies, Warsaw, Poland.

${ }^{21}$ Institute for Experimental Physics, University of Warsaw, Warsaw, Poland.

${ }^{22}$ Faculty of Physics, Warsaw University of Technology, Warsaw, Poland.

${ }^{23}$ Rudjer Boskovic Institute, Zagreb, Croatia.

The elliptic flow for Lambda hyperons and $K_{s}^{0}$ mesons was measured by the NA49 experiment in semicentral $\mathrm{Pb}+\mathrm{Pb}$ collisions at $158 \mathrm{~A} \mathrm{GeV}$. The standard method of correlating particles with an event plane has been used. Measurements of $v_{2}$ near mid-rapidity are reported as a function of centrality, rapidity and transverse momentum. Elliptic flow of $\Lambda$ and $K_{s}^{0}$ particles increases both with the impact parameter and with the transverse momentum. It is compared with $v_{2}$ for pions and protons as well as with various model predictions. The NA49 results are compared with data from NA45/CERES and STAR experiments.

The 3rd edition of the International Workshop - The Critical Point and Onset of Deconfinement July 3-7 2006

Galileo Galilei Institute, Florence, Italy

\footnotetext{
${ }^{*}$ Speaker.
} 


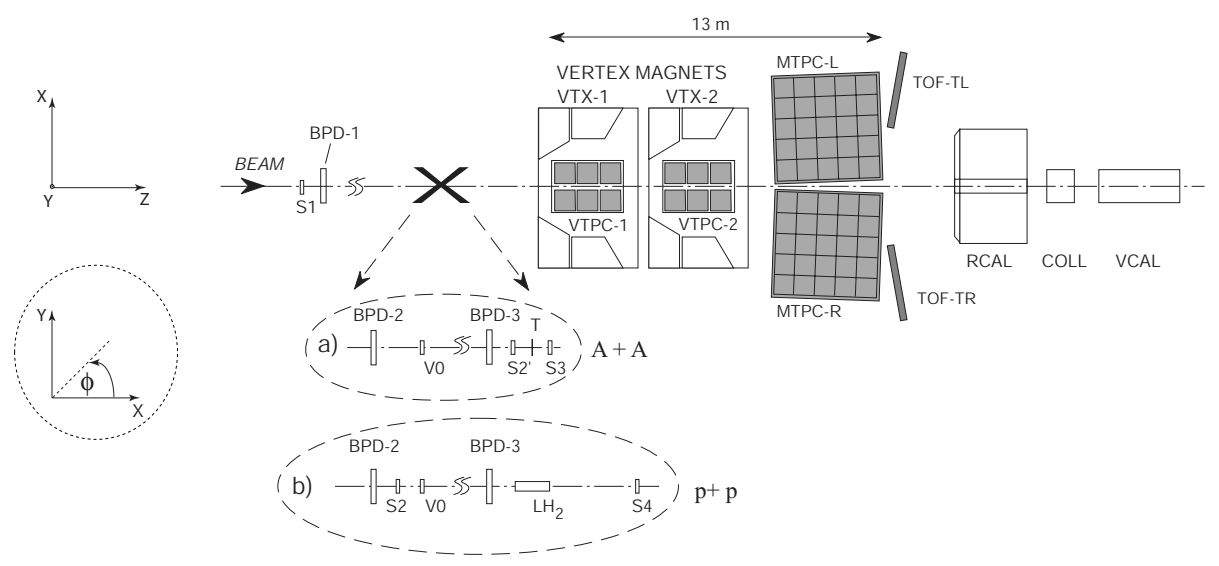

Figure 1: The experimental setup of the NA49 experiment

\section{Introduction}

Elliptic flow has its origin in the spatial anisotropy of the initial reaction volume in non-central collisions and in particle rescatterings in the evolving system which convert the spatial anisotropy into a momentum anisotropy [1]. The spatial anisotropy decreases rapidly because of the fast expansion of the system [2] making the momentum anisotropy measured at the end of this evolution strongly dependent on the matter properties and the effective equation of state (EoS) at the early stage [3, 4]. It is particularly sensitive to the degree of thermalization in the produced particle system. Flow of heavy particles is affected more strongly by changes in the EoS than flow of pions [4, 5, 6]. Moreover, various hadron types are believed to decouple at different times and with different temperatures [7]. Thus the elliptic flow of various particle species allows insight into different stages of the collision. Comparison of measured anisotropies of different particle species with various model calculations, for example hydrodynamical or quark coalescence, provides an important test of various evolution scenarios.

The anisotropic flow parameters measured to date at SPS and lower energies are mainly those of pions and protons [8, 9]. We have extended elliptic flow measurements in $158 \mathrm{~A} \mathrm{GeV} \mathrm{Pb}+\mathrm{Pb}$ $\left(\sqrt{s_{\mathrm{NN}}}=17.2 \mathrm{GeV}\right)$ collisions to $\Lambda$ hyperons and $K_{s}^{0}$ particles to test the validity of the hydrodynamic scenario and check the degree of thermalization at SPS energies.

\section{Experiment and Data}

The NA49 experimental setup is shown in Fig. 1. The main components of the NA49 detector [10] are four large-volume Time Projection Chambers for tracking and particle identification. The TPC system consists of two vertex chambers inside the spectrometer magnets which allow separation of positively and negatively charged tracks and a precise measurement of the particle momenta with a resolution $\sigma(p) / p^{2}=(0.3-7) \times 10^{-4}(\mathrm{GeV} / c)^{-1}$. Two main chambers, placed behind the magnets at both sides of the beam, were optimized for high precision detection of the ionization loss $d E / d x$ with a resolution of 3-6\%. Downstream of the TPCs a veto calorimeter detects projectile spectators and is used for triggering and centrality selection. The data sample consists of $3 \times 10^{6}$ 


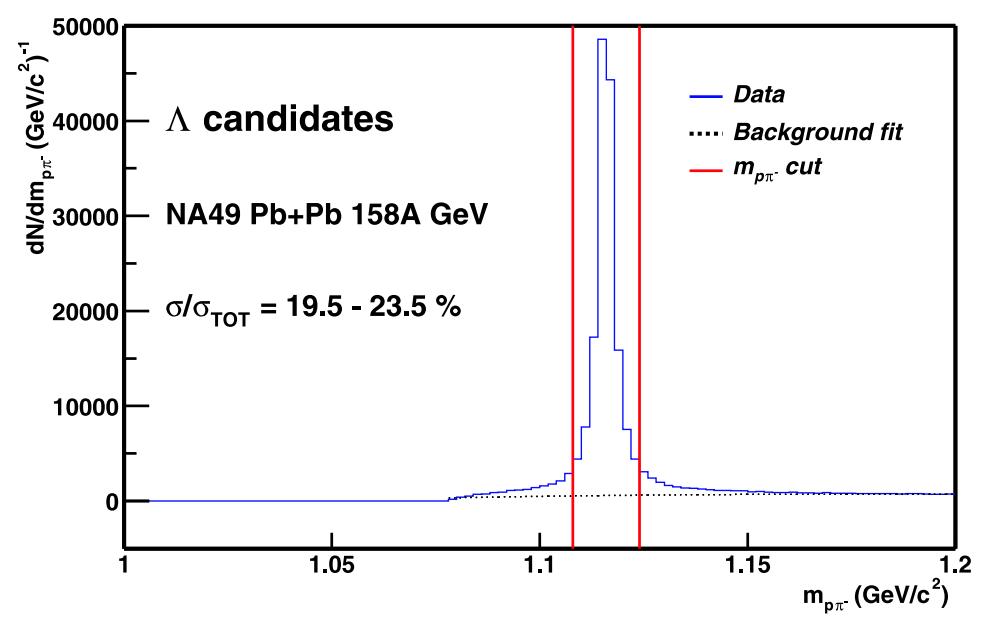

Figure 2: The invariant mass distribution of $\mathrm{p} \pi^{-}$candidate pairs in $\mathrm{Pb}+\mathrm{Pb}$ collisions in the centrality range $\sigma / \sigma_{T O T}=19.5-23.5 \%$. The polynomial parametrization of the background is indicated by the dashed line. Red vertical lines represent invariant mass cuts.

semi-central $\mathrm{Pb}+\mathrm{Pb}$ events after online trigger selection of the $23.5 \%$ most central collisions. The events were divided into three different centrality bins, which correspond to the first three bins used in a previous analysis (see Table 1 in [9]). They are defined by centrality ranges 0-5\% (bin 1), $5-12.5 \%$ (bin 2), and $12.5-23.5 \%$ (bin 3) which correspond to impact parameter ranges which are: 0-3.4 fm (bin 1), 3.4-5.3 fm (bin 2), and 5.3-7.4 fm (bin 3). The measurement in the centrality range $\sigma / \sigma_{T O T}=5-23.5 \%$ (called mid-central) is obtained by averaging the results of bins 2 plus 3 with weights corresponding to the fractions of the total cross section in these bins.

\section{Selection of particles}

The $\Lambda$ hyperon candidates were selected from the sample of $\mathrm{V}^{0}$-track configurations consisting of oppositely charged particles, which include the $\Lambda$ decays into proton and $\pi^{-}$(branching ratio 63.9\%). Geometric and quality criteria ensured that only reliably reconstructed tracks were processed. The identification method [11] relies on the evaluation of the invariant mass distribution and is enhanced by daughter particle identification applying a cut in $d E / d x$ around the expectation value derived from a Bethe-Bloch parametrization. $K_{s}^{0}$ mesons, which decay into $\pi^{+}$and $\pi^{-}$ (branching ratio 68.6\%), were identified in a similar way as $\Lambda$ hyperons. Instead of identification of daughter particles by $d E / d x$ measurement we assigned the proton mass to one of the daughter particles and excluded those pair candidates which give entries near the expected $\Lambda, \bar{\Lambda}$ invariant mass peak, $1.100<m_{p \pi^{-}}, m_{\bar{p} \pi^{+}}<1.132 \mathrm{GeV} / c^{2}$ (compare Fig. 3 (left) and (right)). The yields of $\Lambda$ hyperons and $K_{s}^{0}$ particles are obtained by counting the number of entries in the invariant mass peak above the estimated background as a function of the azimuthal angle $\phi_{\text {lab }}$ with respect to the event plane (see below). The background is estimated from a fit of sum of Lorentz distribution for $K_{s}^{0}$ or $\Lambda$ and a polynomial background (Fig. 2 and Fig. 3). The number of accepted particles in mid-central events is about 740.00 for $\Lambda$ hyperons in the invariant mass window $1.108-1.124 \mathrm{GeV} / c^{2}$ and 

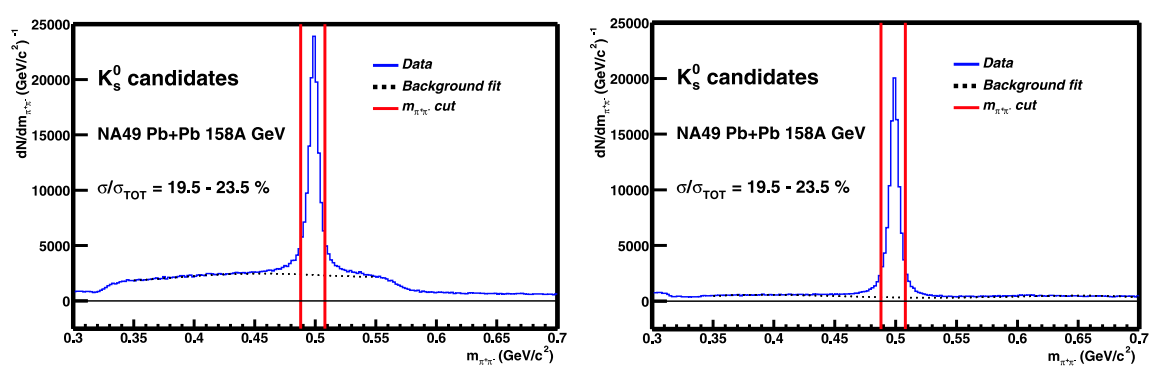

Figure 3: The invariant mass distributions of $\pi^{+} \pi^{-}$candidate pairs in $\mathrm{Pb}+\mathrm{Pb}$ collisions at $\sqrt{s_{N N}}=17.2 \mathrm{GeV}$ in the centrality range $\sigma / \sigma_{T O T}=19.5-23.5 \%$. The distribution is with (left) and without (right) admixture of $p \pi^{-}, \bar{p} \pi^{+}$candidate pairs with an invariant mass near to the expected $\Lambda, \bar{\Lambda}$ hyperon peak (see text for details). The polynomial parametrization of the background is indicated by the dashed line. Red vertical lines represent invariant mass cuts.
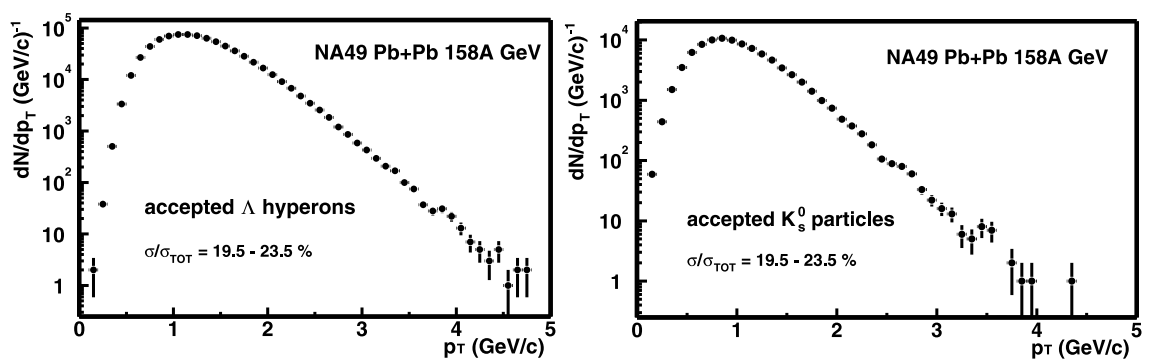

Figure 4: Transverse momentum distributions for $\Lambda$ hyperons (left) and $K_{s}^{0}$ particles (right) measured in the centrality range $\sigma / \sigma_{T O T}=19.5-23.5 \%$ of $\mathrm{Pb}+\mathrm{Pb}$ collisions at $\sqrt{s_{N N}}=17.2 \mathrm{GeV}$.

440.000 for $K_{s}^{0}$ mesons in the invariant mass window $0.488-0.508 \mathrm{GeV} / c^{2}$. The acceptance of $\Lambda$ hyperons covers the range $0.4 \lesssim p_{\mathrm{T}} \lesssim 4.0 \mathrm{GeV} / c$ (see Fig. 4 (left)) and $-1.5 \lesssim y \lesssim 1.0$ and strongly depends on $p_{\mathrm{T}}$ and $y$. The range of transverse momentum of $K_{s}^{0}$ mesons is more narrow $0.2 \lesssim p_{\mathrm{T}} \lesssim 3.5 \mathrm{GeV} / c$ (see Fig. 4(right)). Multiplicative factors were introduced for every $\Lambda$ particle to correct the $\Lambda$ yields for detector and reconstruction efficiency which depends on $p_{\mathrm{T}}$ and $y$. Such an additional correction has not been introduced for $K_{s}^{0}$ mesons so far. $K_{s}^{0}$ elliptic flow data are still very preliminary.

\section{Method}

The elliptic flow analysis is based on the standard procedure outlined in [9, 12] to reconstruct the event plane for each event and the corrections for the event plane resolution. The event plane is an experimental estimator of the true reaction plane and is calculated from the azimuthal distribution of primary charged $\pi$ mesons. Identification of pions is based on energy loss measurements $(d E / d x)$ in the TPCs. To avoid possible auto-correlations, tracks associated with $\Lambda$ or $K_{s}^{0}$ candidates are excluded from the event plane calculation. The method to determine the event plane angle $\Phi_{2 E P}$ 


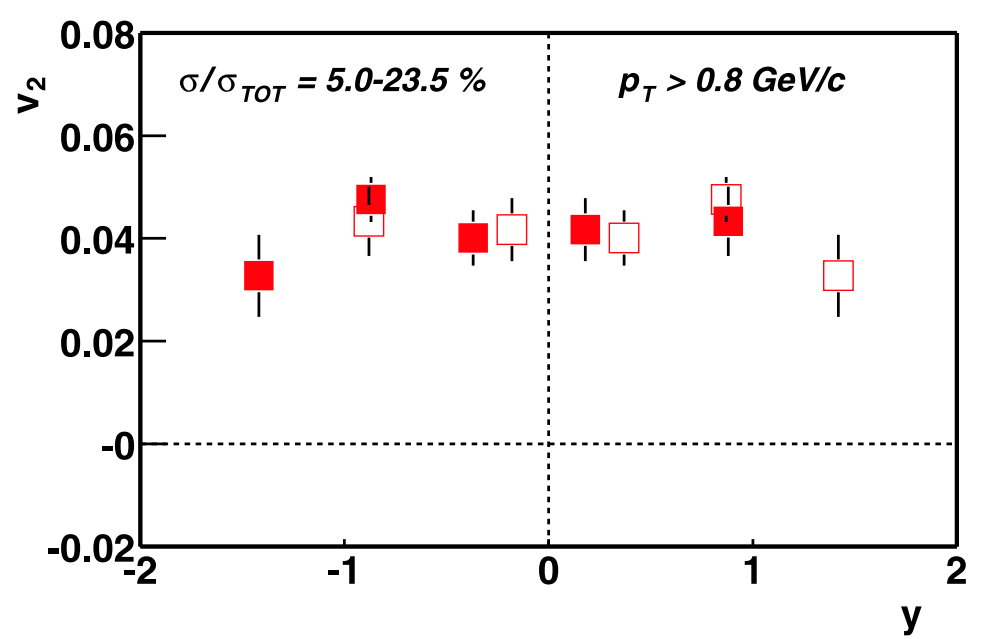

Figure 5: Elliptic flow of $\Lambda$ hyperons measured in mid-central $\mathrm{Pb}+\mathrm{Pb}$ collisions as a function of rapidity. The open points have been reflected about midrapidity.

uses the elliptic flow of pions, according to the formula:

$$
\begin{aligned}
X_{2} & =\sum_{i=1}^{N} p_{\mathrm{T}}^{i}\left[\cos \left(2 \phi_{\mathrm{lab}}^{i}\right)-\left\langle\cos \left(2 \phi_{\mathrm{lab}}\right)\right\rangle\right] \\
Y_{2} & =\sum_{i=1}^{N} p_{\mathrm{T}}^{i}\left[\sin \left(2 \phi_{\mathrm{lab}}^{i}\right)-\left\langle\sin \left(2 \phi_{\mathrm{lab}}\right)\right\rangle\right] \\
\Phi_{2 \mathrm{EP}} & =\tan ^{-1}\left(\frac{Y_{2}}{X_{2}}\right) / 2 .
\end{aligned}
$$

where $X_{2}, Y_{2}$ are the components of the event plane flow vector $\mathbf{Q}_{2}$ and the sums run over accepted charged pion tracks. The acceptance correction is based on the recentering method of [9] which consists of subtracting in Eq. 4.1 the mean values $\left\langle\cos \left(2 \phi_{\text {lab }}\right)\right\rangle$ and $\left\langle\sin \left(2 \phi_{\text {lab }}\right)\right\rangle$. These mean values are calculated in bins of $p_{\mathrm{T}}$ and rapidity for all charged pions in those events which contain at least one $\Lambda$ hyperon or $K_{s}^{0}$ meson candidate. The means were stored in a 3-dimensional matrix of $20 p_{\mathrm{T}}$ intervals, 50 rapidity intervals, and eight centrality bins.

A second level acceptance correction is done by using mixed events. We used 10 mixed events for each real event. Particles for mixed events are randomly selected from different events in the same centrality bin with at least one $\Lambda$ or $K_{s}^{0}$ particle. The final angular distributions were obtained by dividing the real $\Lambda$ and $K_{s}^{0}$ angular distributions by the mixed event distributions to remove the acceptance correlations remaining after recentering. The corrected particle azimuthal distributions are then fitted with a truncated Fourier series:

$$
\begin{aligned}
\frac{d N}{d\left(\phi_{\mathrm{lab}}-\Phi_{2 \mathrm{EP}}\right)}= & \\
\operatorname{const} \times(1 & +v_{2}^{o b s} \cos \left[2\left(\phi_{\mathrm{lab}}-\Phi_{2 \mathrm{EP}}\right)\right] \\
& \left.+v_{4}^{o b s} \cos \left[4\left(\phi_{\mathrm{lab}}-\Phi_{2 \mathrm{EP}}\right)\right]\right) .
\end{aligned}
$$



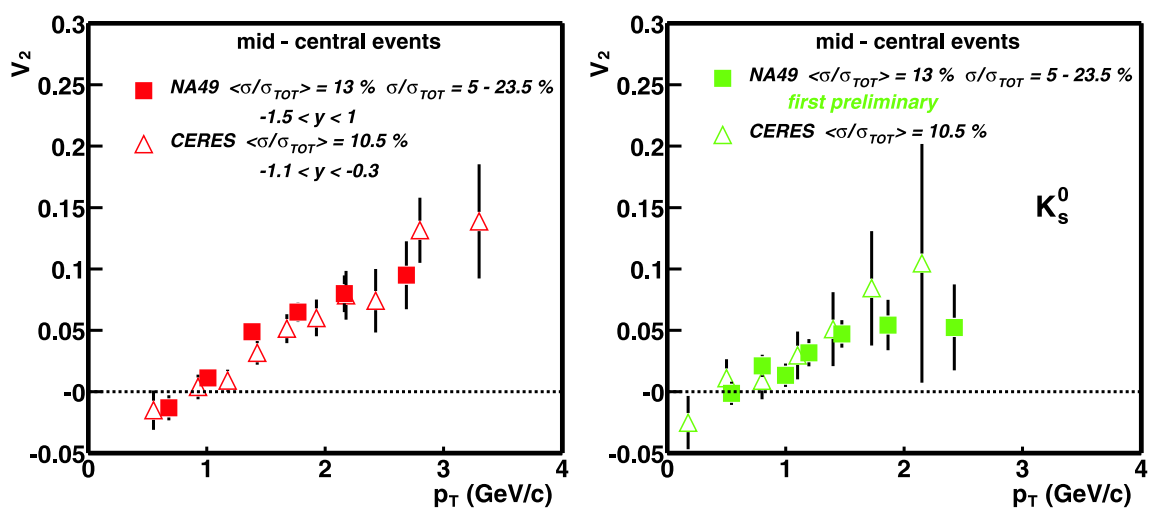

Figure 6: Elliptic flow of $\Lambda$ hyperons (left) and $K_{s}^{0}$ mesons measured by $\mathrm{NA} 49$ in $\mathrm{Pb}+\mathrm{Pb}$ and NA45/CERES in $\mathrm{Pb}+\mathrm{Au}$ mid-central collisions as a function of transverse momentum.
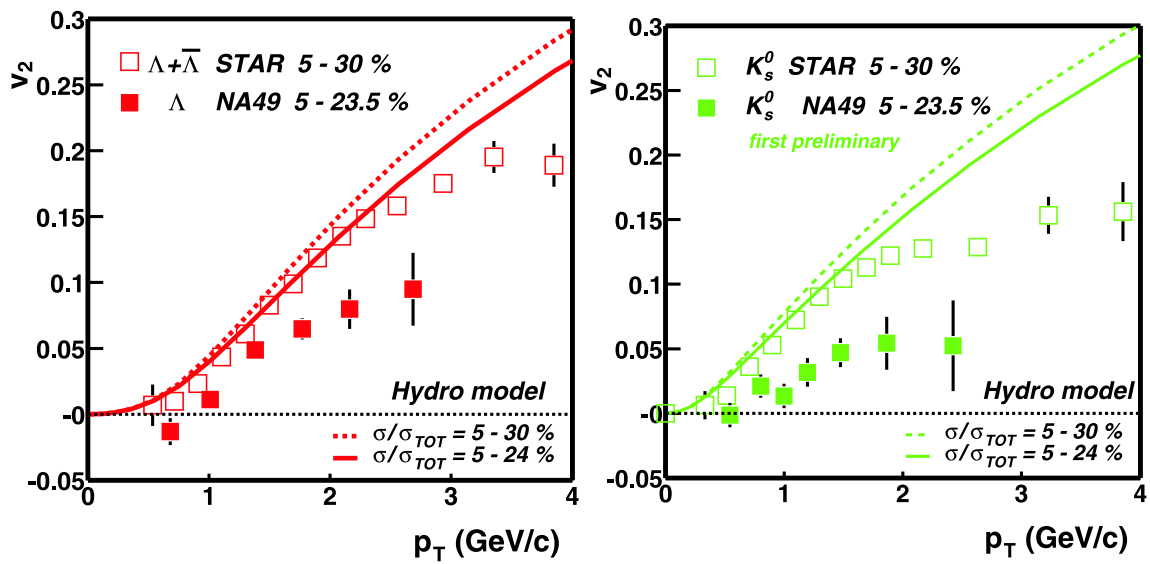

Figure 7: Dependence of $v_{2}$ on $p_{\mathrm{T}}$ for $\Lambda$ hyperons (left) and $K_{s}^{0}$ mesons (right) measured at $\sqrt{s_{N N}}=17.2$ $\mathrm{GeV}$ and $\sqrt{s_{N N}}=200 \mathrm{GeV}$ in comparison to a hydrodynamical calculation at $\sqrt{s_{N N}}=200 \mathrm{GeV}$ for two centrality ranges.

The elliptic flow $v_{2}$ is evaluated by dividing the observed anisotropy $v_{2}^{o b s}$ by the event plane resolution $R$ :

$$
v_{2}=\frac{v_{2}^{o b s}}{R}
$$

The event plane resolution,

$$
R=\left\langle\cos \left[2\left(\Phi_{2 \mathrm{EP}}-\Phi_{\mathrm{r}}\right)\right]\right\rangle=\sqrt{2\left\langle\cos \left[2\left(\Phi_{2 \mathrm{EP}}^{a}-\Phi_{2 \mathrm{EP}}^{b}\right)\right]\right\rangle},
$$

is calculated from the correlation of two planes $\left(\Phi_{2 \mathrm{EP}}^{a}, \Phi_{2 \mathrm{EP}}^{b}\right)$ for random sub-events with equal multiplicity. The results are $R=0.27,0.34$ and 0.40 for centrality bins 1,2 and 3, respectively. The uncertainty due to the background subtraction, event plane resolution and the mixed event corrections are added to the total error. The observed hexadecupole anisotropy $v_{4}^{o b s}$ is consistent with zero within statistical errors. 


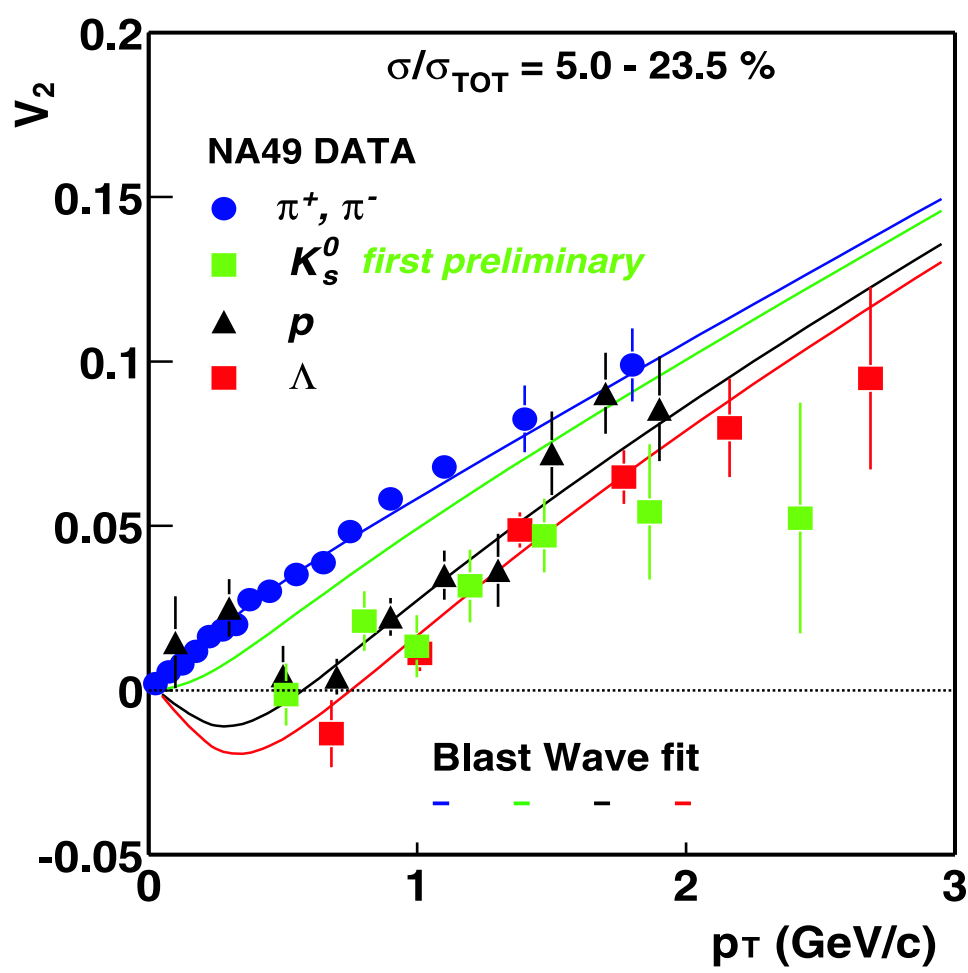

Figure 8: Plot of $v_{2}$ of charged pions, protons, $\Lambda$ hyperons and $K_{s}^{0}$ mesons as a function of $p_{T}$ in mid-central $\mathrm{Pb}+\mathrm{Pb}$ collisions at $\sqrt{s_{N N}}=17.2 \mathrm{GeV}$. The solid lines represent results of a blast wave fit.

\section{Results}

Fig. 5 shows the $p_{\mathrm{T}}$ averaged elliptic flow obtained from all identified $\Lambda$ hyperons without $p_{\mathrm{T}}$ cuts. It exhibits no significant dependence on rapidity as was also observed for protons (see Fig. 6 of [9]) in mid-central events. The full rapidity range of the data in Fig. 5 was used to study $v_{2}$ as a function of $p_{\mathrm{T}}$. The $p_{\mathrm{T}}$ dependence of rapidity-averaged $\Lambda$ and $K_{s}^{0}$ elliptic flow is shown in Fig. 6(left) and Fig. 6(right) respectively in comparison to CERES data[13]. The NA49 $v_{2}$ parameter significantly increases with transverse momentum and agrees with CERES results in $\mathrm{Pb}+\mathrm{Au}$ mid-central collisions at the top SPS energy. Fig. 7 shows a comparison of $v_{2}\left(p_{\mathrm{T}}\right)$ of $\Lambda$ hyperons (left panel) and $K_{s}^{0}$ mesons (right panel) for mid-central events measured by the NA49 and STAR experiments [14]. At SPS energy the elliptic flow grows linearly with $p_{\mathrm{T}}$ up to $\sim 2 \mathrm{GeV} / \mathrm{c}$, but the increase is more pronounced at RHIC than at SPS energy. It should be noted that RHIC mid-central data have been measured in the centrality range $\sigma / \sigma_{\mathrm{TOT}}=5-30 \%$ while SPS events are somewhat more central. The effect of different centrality ranges has been estimated by hydrodynamic calculations [15] at RHIC energy for the slightly different centrality bins of NA49 and STAR. As shown by the corresponding curves in Fig. 7 this explains only partly the difference between both measurements. A comparison of $v_{2}\left(p_{\mathrm{T}}\right)$ for pions, protons, $\Lambda$ hyperons and $K_{s}^{0}$ mesons as measured by the NA49 experiment in mid-central events is displayed in Fig. 8. The values for pions and protons 

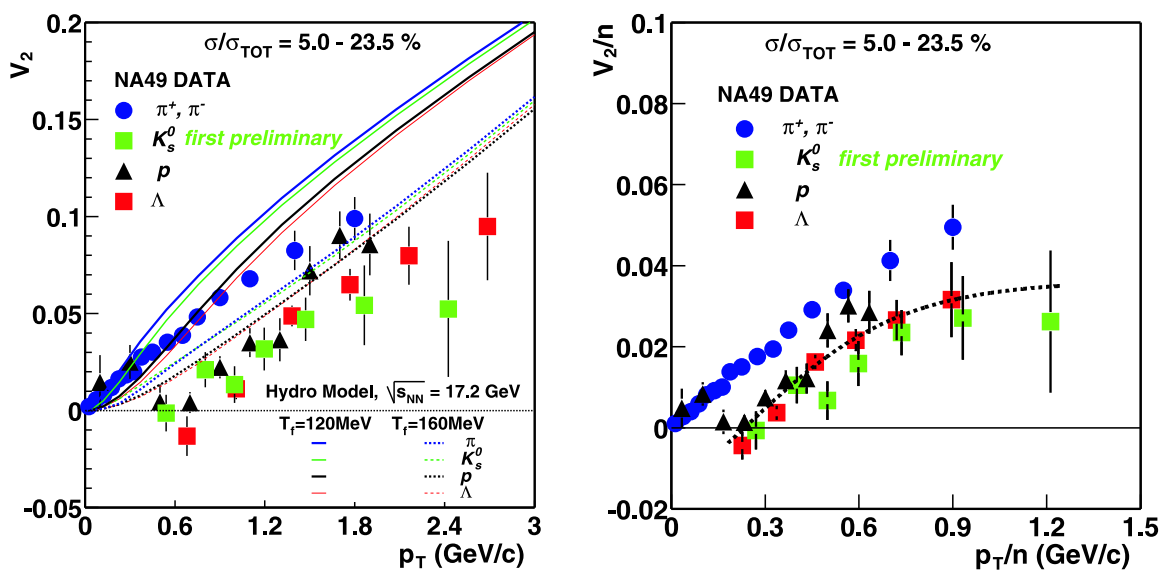

Figure 9: Left: $v_{2}\left(p_{T}\right)$ of charged pions, protons, $\Lambda$ hyperons and $K_{s}^{0}$ mesons from $158 \mathrm{~A} \mathrm{GeV} \mathrm{Pb}+\mathrm{Pb}$ midcentral collisions. Hydrodynamic model calculations at $\sqrt{s_{N N}}=17.2 \mathrm{GeV}$ are shown as solid $\left(T_{f}=120 \mathrm{MeV}\right)$ and dashed $\left(T_{f}=160 \mathrm{MeV}\right.$ ) lines (see text for details). Right: $v_{2}$ scaled by the number of quark as a function of scaled $p_{T}$. All data are from $158 \mathrm{~A} \mathrm{GeV} \mathrm{Pb}+\mathrm{Pb}$ mid-central collisions. The dashed line is the scaled result of the fit to $\mathrm{p}, \Lambda$ and $K_{s}^{0}$.

were obtained as the cross section weighted averages of the measurements published in [9] for the appropriate centrality range. As seen in Fig. 8 the elliptic flow grows linearly with $p_{\mathrm{T}}$ for all particle species but the rise for pions starts from $p_{\mathrm{T}}$ close to zero while for protons, $\Lambda$ and $K_{s}^{0}$ mesons it starts from $p_{\mathrm{T}} \approx 0.5 \mathrm{GeV} / c$. The elliptic flow for pions is significantly larger than that for heavier particles although at $p_{\mathrm{T}} \approx 2 \mathrm{GeV} / c$ the flow becomes similar for all particle species. Except for $K_{s}^{0}$ mesons the measurements are reproduced by blast wave fits [5, 16, 17] (curves in Fig. 8) with the following parameters: freeze-out temperature $T=95 \mathrm{MeV}$, mean transverse expansion rapidity $\rho_{0}=0.85$, its second harmonic azimuthal modulation amplitude $\rho_{2}=0.021$ and the variation in the azimuthal density of the source elements $s_{2}=0.035$. In Fig. 9(left) the measured values of $v_{2}$ are compared to hydrodynamical model calculations [18] assuming a first-order phase transition to a QGP at the critical temperature $T_{\mathrm{c}}=165 \mathrm{MeV}$. With the freeze-out temperature $T_{\mathrm{f}}=120 \mathrm{MeV}$ tuned to reproduce particle spectra, the model calculations significantly overestimate the SPS results for semi-central collisions (full curves in Fig. 9(left)) in contradiction to predictions at RHIC energy which agree with data quite well for $p_{\mathrm{T}} \lesssim 2 \mathrm{GeV} / c$ [14]. The discrepancy at SPS may indicate a lack of complete thermalisation or a viscosity effect. On the other hand, the model reproduces qualitatively the characteristic hadron-mass ordering of elliptic flow. Thus the data support the hypothesis of early development of collectivity. The calculation from the same model with a higher temperature $T_{\mathrm{f}}=160 \mathrm{MeV}$ exhibits better agreement with the $\Lambda$ flow data (dotted curves in Fig. 9(left)). Unfortunately, the model does not simultaneously reproduce the $m_{\mathrm{T}}$ spectra with such a high freeze-out temperature. A partial solution of this problem can be found by coupling a hadronic rescattering phase to the hydrodynamical evolution and hadronisation [4].

Coalescence models predict that $v_{2}$ will approximately scale with the number $n$ of constituent quarks. When plotting at the similarly scaled value $p_{\mathrm{T}} / n$ results for all hadrons are expected to 
fall on an universal. This prediction agrees well with RHIC data in the intermediate transverse momentum region $p_{\mathrm{T}} / n>0.7 \mathrm{GeV} / c$ [14]. The universal curve for all hadrons is expected to represent the momentum-space anisotropy of constituent quarks prior to hadron formation. The naive coalescence model roughly agrees with our proton, $\Lambda$ and $K_{s}^{0}$ at higher $p_{\mathrm{T}}$ values ( Fig.9(right)) although the $p_{\mathrm{T}}$ range of accepted particles is too narrow and error bars are to large to draw a clear conclusion. In more realistic quark coalescence model the resonance decays and quark momentum distribution in hadrons can lead to higher $v_{2}$ for pions and generally deviations of meson elliptic flow from the scaling behavior as observed on Fig. 9(right) and also seen by the STAR experiment.

\section{Acknowledgments}

This work was supported by the US Department of Energy Grant DE-FG03-97ER41020/A000, the Bundesministerium fur Bildung und Forschung 06F-137, Germany, the Virtual Institute VI-146 of Helmholtz Gemeinschaft, Germany, the Polish State Committee for Scientific Research (1 P03B 006 30, 1 P03B 097 29, 1 P03B 121 29, 1 P03B 127 30), the Hungarian Scientific Research Foundation (T032648, T032293, T043514), the Hungarian National Science Foundation, OTKA, (F034707), the Polish-German Foundation, the Korea Science \& Engineering Foundation (R012005-000-10334-0) and the Bulgarian National Science Fund (Ph-09/05).

\section{References}

[1] J. Y. Ollitrault, Nucl. Phys. A 638, 195 (1998).

[2] P. F. Kolb, J. Sollfrank and U. W. Heinz, Phys. Rev. C 62, 054909 (2000).

[3] J. Y. Ollitrault, Phys. Rev. D 46, 229 (1992); H. Sorge, Phys. Rev. Lett. 82, 2048 (1999).

[4] D. Teaney, J. Lauret and E. V. Shuryak, Phys. Rev. Lett. 86, 4783 (2001) and nucl-th/0110037.

[5] P. Huovinen, P. F. Kolb, U. W. Heinz, P. V. Ruuskanen and S. A. Voloshin, Phys. Lett. B 503, 58 (2001).

[6] R. Snellings [STAR Collaboration], Heavy Ion Phys. 21, 237 (2004)

[7] H. van Hecke, H. Sorge and N. Xu, Phys. Rev. Lett. 81, 5764 (1998).

[8] N. Bastid et al. [FOPI Collaboration], Nucl. Phys. A 622, 573 (1997); A. Andronic et al. [FOPI Collaboration], Nucl. Phys. A 679, 765 (2001); J. Barrette et al. [E877 Collaboration], Phys. Rev. C 55, 1420 (1997); H. Appelshauser et al. [NA49 Collaboration], Phys. Rev. Lett. 80, 4136 (1998).

[9] C. Alt et al. [NA49 Collaboration], Phys. Rev. C 68, 034903 (2003).

[10] S. V. Afanasiev et al. [NA49 Collaboration], Nucl. Intr. Meth. A 430, 210 (1999).

[11] H. Appelshauser et al. [NA49 Collaboration], J. Phys. G 25, 469 (1999); L.S. Barnby, Ph.D. Thesis, University of Birmingham (1999).

[12] S. A. Voloshin and A. M. Poskanzer, Phys. Lett. B 474, 27 (2000).

[13] J. Milosěvić et al. [NA45 Collaboration], nucl-ex/0510057 (2005), nucl-ex/0606020 (2006), and private communication.

[14] J. Adams et al. [STAR Collaboration], Phys. Rev. Lett. 92, 052302 (2004). 
[15] P. Huovinen, Nucl. Phys. A 761, 296 (2005), and private communication.

[16] C. Adler et al. [STAR Collaboration], Phys. Rev. Lett. 87, 182301 (2001).

[17] F. Retiere and A. M. Lisa, Phys. Rev. C 70, 044907 (2004).

[18] P. Huovinen, private communication (2005), $T_{\mathrm{c}}=165 \mathrm{MeV}, T_{\mathrm{f}}=120,160 \mathrm{MeV}$, EoS=Q. 